\title{
1 Widespread microbial mercury methylation genes in the global ocean
}

2 Emilie Villar $^{1,2}$, Lea Cabrol ${ }^{1 *}$, Lars-Eric Heimbürger-Boavida ${ }^{1 *}$

$4 \quad{ }^{1}$ Aix Marseille Université, Univ Toulon, CNRS, IRD, Mediterranean Institute of Oceanography

5 (MIO) UM 110, 13288, Marseille, France

$6 \quad{ }^{2}$ Sorbonne Université, Université Pierre et Marie Curie - Paris 6, CNRS, UMR 7144 (AD2M),

7 Station Biologique de Roscoff, Place Georges Teissier, CS90074, Roscoff, 29688 France

$8 \quad$ *Both authors contributed equally to this work.

9 Corresponding Author: Léa Cabrol, lea.cabrol@mio.osupytheas.fr

\section{Abstract}

12 Methylmercury is a neurotoxin that bioaccumulates from seawater to high concentrations in marine fish, putting human and ecosystem health at risk. High methylmercury levels have been found in the oxic subsurface waters of all oceans, yet only anaerobic microorganisms have been identified so far as efficient methylmercury producers in anoxic environments.

16 The microaerophilic nitrite oxidizing bacteria Nitrospina has been previously suggested as a

17 possible mercury methylator in Antarctic sea ice. However, the microorganisms processing

18 inorganic mercury into methylmercury in oxic seawater remain unknown. Here we show

19 metagenomic evidence from open ocean for widespread microbial methylmercury

20 production in oxic subsurface waters. We find high abundances of the key mercury

21 methylating genes $h g c A B$ across all oceans corresponding to taxonomic relatives of known

22 mercury methylators from Deltaproteobacteria, Firmicutes and Chloroflexi. Our results 
23 identify Nitrospina as the predominant and widespread key player for methylmercury

24 production in the oxic subsurface waters of the global ocean.

25

26

27

28

\section{Introduction}

Human activities release 2500 tons of inorganic mercury $(\mathrm{Hg})$ every year and have added 55 000 tons of $\mathrm{Hg}$ to the global ocean since the industrial revolution ${ }^{1}$. Humans are exposed to $\mathrm{Hg}$ in the form of methylmercury (MeHg), mainly through the consumption of marine fish. The Minamata Convention (www.mercuryconvention.org) aims to protect human health from the adverse effects of $\mathrm{Hg}$ via the reduction of anthropogenic, inorganic $\mathrm{Hg}$ emissions. To understand the efficacy and time-scales of lowered $\mathrm{Hg}$ emissions to reduce fish $\mathrm{MeHg}$ levels, we must fully understand the origin of marine MeHg. Microorganisms play a central role in $\mathrm{Hg}$ transformations. We must identify the $\mathrm{Hg}$ methylating microbes and the factors controlling their distribution in order to better constrain $\mathrm{MeHg}$ production in the global ocean.

As the only cultured microbes known to produce $\mathrm{MeHg}$ to date are anaerobic, research focused for many years on a MeHg source in anoxic marine sediments ${ }^{2-5}$. However, several lines of independent evidence speak in favour of in situ MeHg production in oxic seawater as the main source of fish MeHg. Recent large scale oceanographic expeditions found subsurface MeHg maxima in every ocean basin ${ }^{4,6}$. The proportion of MeHg to inorganic $\mathrm{Hg}$ throughout the oxic seawater column is higher than those found in anoxic sediments.

Laboratory experiments show that $\mathrm{Hg}$ methylation can occur in anaerobic microniches that occur within sinking particles in oxic waters ${ }^{7}$. Bianchi et al. ${ }^{8}$ provide compelling evidence that anaerobic microbes thrive in anoxic microenvironments of sinking particulate organic matter. Independently, incubation experiments with isotopically labelled $\mathrm{Hg}$ spikes show 
47 significant in situ $\mathrm{Hg}$ methylation in oxic seawater ${ }^{9}$. Additional evidence stems from $\mathrm{Hg}$

48 stable isotope signatures of marine fish, that can only be explained if $60-80 \%$ of the MeHg is

49 produced in open ocean subsurface waters ${ }^{10}$. Lastly, a pioneering study found a compound

50 specific $\delta^{13} \mathrm{C}$ signature of fish tissue MeHg similar to algal $\delta^{13} \mathrm{C}$, suggesting that $\mathrm{MeHg}$ is

51 produced in the open ocean water column ${ }^{11}$.

52 A major breakthrough has been made with the discovery of two key genes, $h g c A$ and $h g c B$,

53 that control $\mathrm{Hg}$ methylation in model anaerobic $\mathrm{Hg}$-methylators ${ }^{5}$. The presence of the hgcAB

54 operon predicts $\mathrm{Hg}$ methylation capacity in diverse microorganisms ${ }^{2}$. A screening of publicly

55 available microbial metagenomes found the $h g c A B$ genes in nearly all anaerobic

56 environments, but the study only rarely detected the genes in pelagic marine water column

57 metagenomes in the open ocean ${ }^{12}$. In antarctic sea ice a marine microaerophilic nitrite

58 oxidizing bacterium belonging to the Nitrospina genus has been recently identified as a

59 potential $\mathrm{Hg}$ methylator with $\mathrm{HgcA}$-like proteins ${ }^{13}$. We aim to resolve the paradox between

60 the wealth of geochemical evidence for in situ MeHg production and the absence of known

61 anaerobic $\mathrm{Hg}$ methylators in the open ocean. Metagenomic data from 243 Tara Oceans

62 samples from 68 open ocean locations covering most ocean basins was analysed to generate

63 an ocean microbial reference gene catalog ${ }^{14}$. We screened the Tara Oceans metagenomes

64 for the presence of the key hgcA methylating gene and provide compelling evidence on the

65 potential key players producing $\mathrm{MeHg}$ in the open ocean.

66 Results and Discussion

67 Identification of HgcAB homologs in the ocean gene catalog

68 Ten $h g c A$ and $5 h g c B$ homolog genes were identified in the Ocean Microbial Reference Gene

69 Catalog ${ }^{14}$ (OM-RGC), 6 scaftigs presenting simultaneously hgcA and hgcB (Fig. 1, 
70 Supplementary Table 1). Alignment of HgcA sequences revealed 7 sequences with the

71 conserved NVWCAA motif ${ }^{5}$ and one sequence with the modified NIWCAA motif on the 'cap

72 helix' region. Mutation experiments previously showed that the structure of the putative

73 'cap helix' region harbouring Cys93 is crucial for methylation function ${ }^{15}$. Two HgcA

74 sequences were truncated (OM-RGC.v1.019516181, OM-RGC.v1.015822836), preventing

75 inspection of their conserved motif, but they could be unequivocally assigned to HgcA

76 sequences based on their phylogenetic placement and high similarity with known HgcA

77 sequences (Fig. 2). The 5 HgcB sequences presented the conserved motif ECGAC 5

78 (Supplementary Table 1).

a

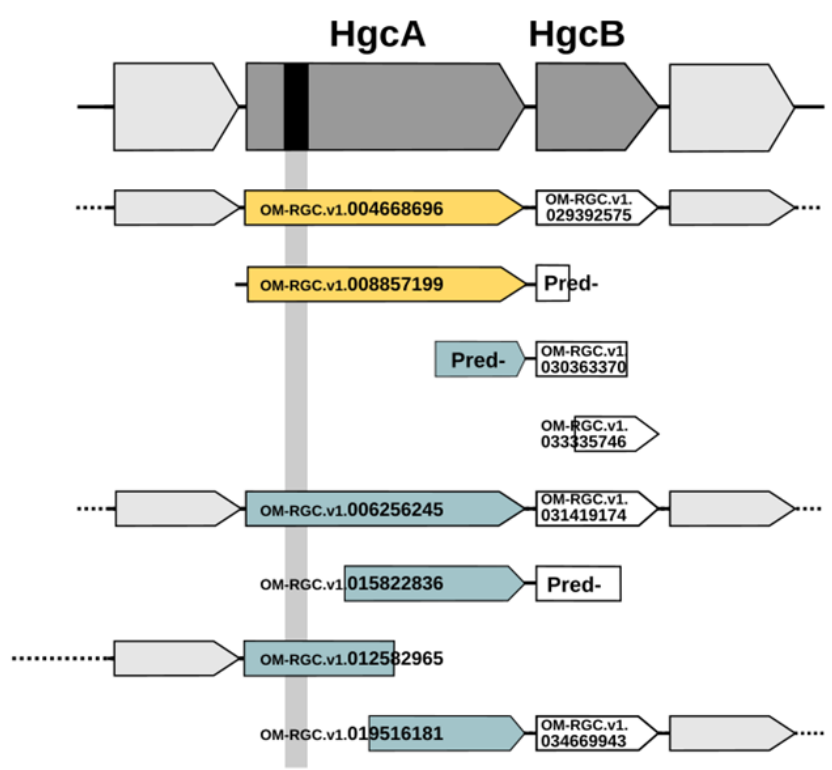

b

HgcAB

CEPV01040748.1

CEQE01294663.1

Z

CESQ01297541.1

CEUC01222703.1

CEUM01136369.1

CEVA01176593.1

CEWJ01142494.1

CEWJ01269211.1

MerR1 DUF169 UbiE HgcA YHS

HgcA-like

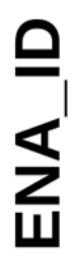

CETA01019545.1

CETS01151954.1

CESC01027225.1

CESG01043795.1
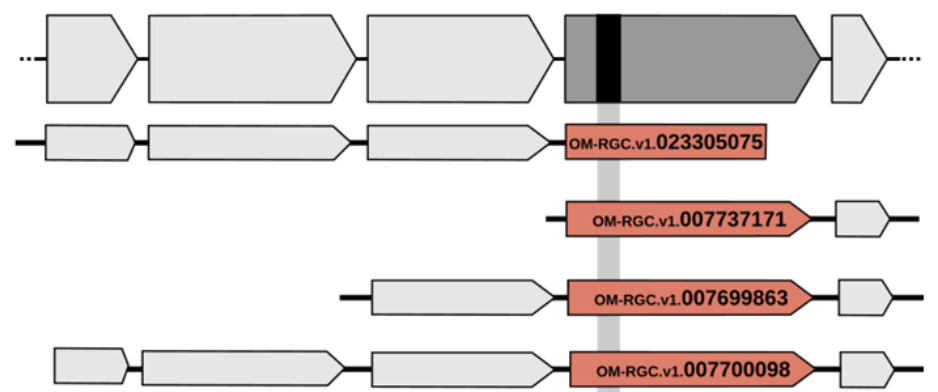

80 Figure 1 | The genomic context of the HgcA orthologs. a, HgcAB operon. b, HgcA-like proteins. The

8112 retrieved scaftigs are identified by their ENA_ID on the left of the figure. The solid lines represent 
82 the extent of the scaftig sequence and the dashed lines indicate that the scaftig sequence is longer

83 than the represented part. The location of the conserved motif is indicated on the HgcA box by a

84 black bar. When present in Tara Oceans samples, the corresponding gene identifier is indicated on

85 the bar for $\mathrm{HgcA}$ and $\mathrm{HgcB}$, or indicated as (Pred-) if the gene was incomplete and the protein

86 sequence was partially predicted. The colour of the HgcA boxes refers to the biogeographical

87 clustering as defined in Fig.3 (Cluster 1 in blue, Cluster 2 in yellow, Cluster 3 in red). For Cluster 3

88 sequences (assigned to Nitrospina), the genomic context was enlarged to show the closest sequences

89 (MerR1: mercuric resistance operon regulatory protein, UbiE: Ubiquinone/menaquinone

90 biosynthesis C-methyltransferase, DUF169: Hypothetical protein with DUF 169 motif, YHS:

91 Hypothetical protein with YHS domain).

92

HgcA sequences found in the Tara Oceans assemblies covered nearly all known $\mathrm{Hg}$

methylators

Phylogenetic placement of the 10 sequences found in the Tara Oceans assemblies covered

nearly all known Hg methylators (Fig. 2). Four sequences (OM-RGC.v1.007700098, OM-

RGC.v1.007737171, OM-RGC.v1.023305075, OM-RGC.v1.007699863) were closely related to

the HgcA-like proteins described by Gionfriddo et al. ${ }^{13}$ for Nitrospina sp. The Nitrospinae

phylum has been described as a distinct phylogenetic group of lithoautotrophic nitrite

oxidizing bacteria exclusively found in marine environments ${ }^{16}$, particularly abundant in oxygen-deficient zones ${ }^{17}$.

The remaining 6 HgcA sequences were distributed between Deltaproteobacteria, Firmicutes and Chloroflexi phyla. Within Deltaproteobacteria, three orders were represented, namely

106 piezophilic sulfate-reducer bacteria (SRB) previously isolated from marine sediment ${ }^{18}$. It

107 belongs to the Desulfovibrionales order, which contains several members with confirmed

108 Hg-methylating capacity, such as the model species Desulfovibrio desulfuricans with 
109 exceptionally high $\mathrm{Hg}$-methylation rates, isolated from estuarine sediments ${ }^{19}$. OM-

110 RGC.v1.019516181 and OM-RGC.v1.012582965 belonged to Desulfobacterales, a well-known

111 order of SRB containing efficient $\mathrm{Hg}$-methylators such as Desulfobulbus propionicus and

112 Desulfococcus multivorans. Finally, OM-RGC.v1.004668696 belonged to

113 Syntrophobacterales. The closest relative of OM-RGC.v1.004668696 with strong confirmed

114 methylation potential was the non-SRB obligate syntroph Syntrophus aciditrophicus ${ }^{2}$.

115 Syntrophic bacteria are important Hg-methylators in low-sulfate ecosystems ${ }^{20,21}$, where

116 they degrade $\mathrm{OM}$ in association with $\mathrm{H}_{2}$-consuming microorganisms such as sulfate-

117 reducers, iron-reducers and methanogens.

118 Within Firmicutes, OM-RGC.v1.015822836 was tightly related to HgcA from recently isolated

119 human gut bacteria Khelaifiella in the Clostridiales order ${ }^{22}$. Their closest relative with

120 confirmed methylation potential is the non-SRB Dethiobacter alkaliphilus, with low to

121 moderate Hg-methylation capacity ${ }^{2}$.

122 OM-RGC.v1.008857199 was related to Chloroflexi, a phylum for which several hgcAB-

123 carriers have been identified, but for which experimental confirmation of $\mathrm{Hg}$ methylation

124 capacity is still needed. This sequence clusters tightly with HgcA from Dehalococcoides

125 mccartyi, which has been reported as a potential methylator, albeit in minor abundance, in

126 freshwater marshes ${ }^{20}$. These two sequences are separated from other Chloroflexi HgcA

127 sequences and more closely related to HgcA sequences from Syntrophobacterales, showing

128 that the taxonomy and the HgcA-phylogeny are not always congruent. The phylogenetically

129 irregular distribution of $h g c A$ can be an indication of horizontal gene transfers (HGT) and/or

130 gene deletions in response to stress, suggesting the prevalent influence of environment on

131 Hg-methylation ability ${ }^{23}$. 
bioRxiv preprint doi: https://doi.org/10.1101/648329; this version posted May 24, 2019. The copyright holder for this preprint (which was not certified by peer review) is the author/funder, who has granted bioRxiv a license to display the preprint in perpetuity. It is made available under aCC-BY-NC-ND 4.0 International license.

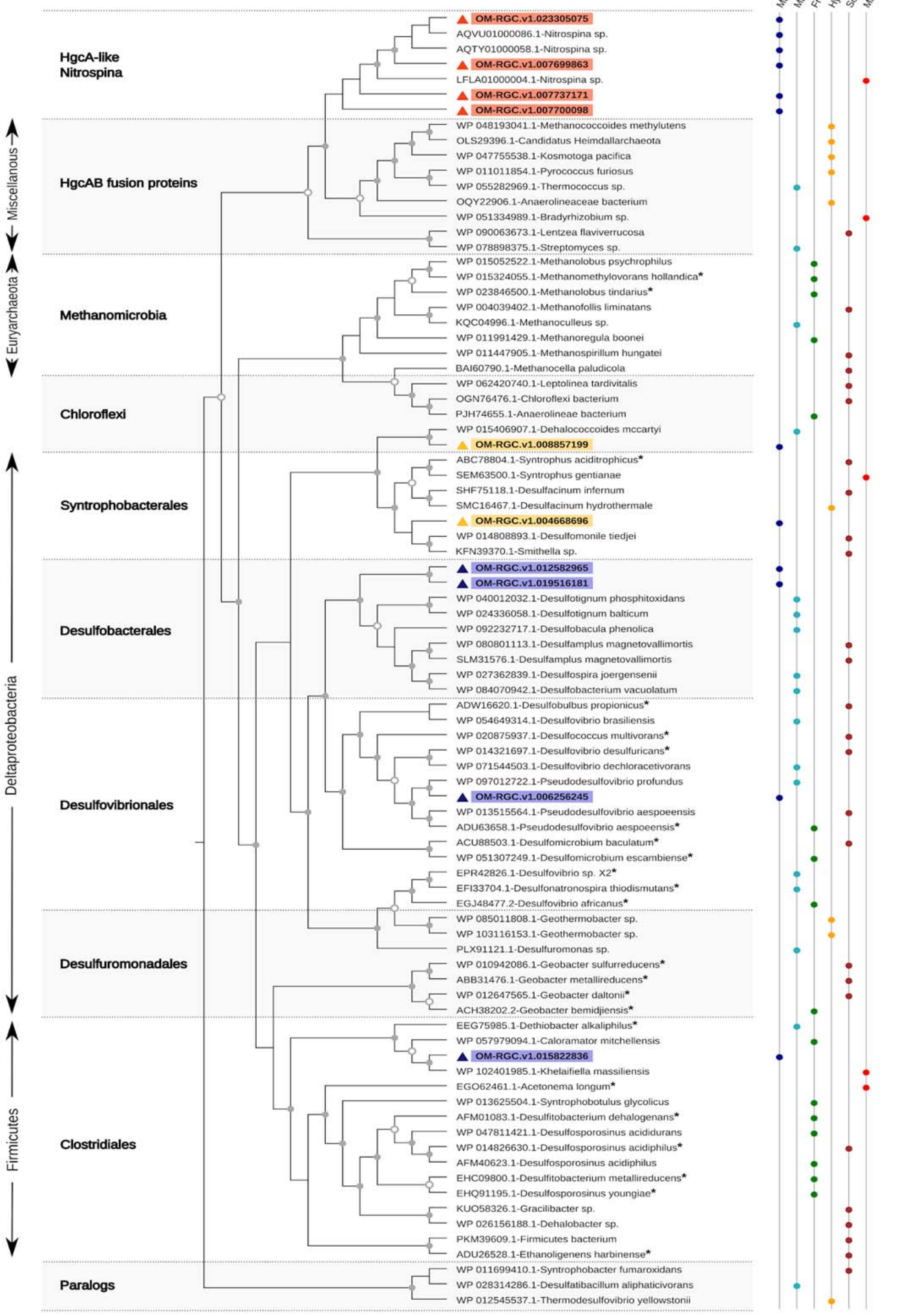

132 
133 Figure 2 | Phylogenetic tree of HgcA homolog sequences found in the Tara Oceans assemblies

134 Maximum likelihood phylogenies were inferred using PhyML Best AIC Tree with the best model of

135 sequence evolution Blosum62+l+G+F. Branch support was calculated using the non-parametric

136 Shimodaira-Hasegawa-like approximate likelihood ratio test. The triangle colour refers to the

137 biogeographical clustering of the HgcA sequences retrieved from Tara Oceans assemblies, as defined

138 in Fig.3 (Cluster 1 in blue, Cluster 2 in yellow, Cluster 3 in red). The tree was rooted using 3

139 paralogues from confirmed non-Hg methylating bacteria. Sequences from experimentally confirmed

140 mercury methylators were indicated with an asterisk. Support values using 1,000 resamples are

141 shown when $>50$ and coloured squares indicate the isolation source.

142

143 Among the $10 \mathrm{HgcA}$ sequences found in the gene ocean catalogue, none was affiliated to

144 methanogenic Archaea. Even if the co-existence of methanogens and sulfate-reducers has

145 been evidenced in marine sediments ${ }^{24}$, sulfate reduction usually outcompetes

146 methanogenesis in seawater under non-limiting sulfate concentrations ${ }^{25}$. Our results thus

147 show that $\mathrm{Hg}$-methylators in the ocean span a large taxonomic diversity, not limited to

148 sulfate-reducing bacteria.

\section{Biogeography distinguishes three groups of putative marine $\mathrm{Hg}$ methylators}

151 Once clearly identified and phylogenetically assigned, the biogeographic distribution

152 patterns of $h g c A$ was evaluated. The $10 \mathrm{HgcA}$ sequences were identified in 77 samples out

153 of the 243 available Tara Oceans metagenomes and were clearly distributed in three clusters

154 according to their abundance patterns (Fig. 3). The biogeographic clustering was consistent

155 with the HgcA-phylogeny.

156 Cluster 1 gathered Desulfobacterales, Clostridiales and Desulfovibrionales HgcA sequences,

157 exclusively present in 23 oxic surface waters $\left(<120\right.$ m-depth, $\left.>10 \mu \mathrm{M}_{\mathrm{O} 2}\right)$. Highest

158 abundances were found in the photic zone of the Pacific Ocean, especially in the area

159 surrounding the Marquesas Islands. This region is characterized by extensive plankton 
160 blooms triggered by a physico-chemical phenomenon called Island Mass Effect related to

161 iron fertilization. In this Cluster, the HgcA sequence OM-RGC.v1.006256245 related to the

162 Desulfovibrionales order (containing most of the experimentally confirmed Hg-methylators)

163 was the most frequent and abundant in the 23 oxic samples.

164 The phylogenetic placement of the two sequences grouped in Cluster 2 is poorly supported.

165 The most abundant sequence was related to Smithella and Desulfomonile tiedjei

166 (Syntrophobacterales) while the other one was close to Chloroflexi (Fig. 2, Supplementary

167 Table 2). HgcA sequences from Cluster 2 were identified in 15 surface and subsurface

168 samples, mostly in suboxic waters : sequences found in samples with oxygen concentration

169 below $10 \mu \mathrm{M}$ accounted for $98 \%$ of total Cluster 2 abundances (Supplementary Figure 2).

170 The highest abundances of Cluster $2 \mathrm{HgcA}$ sequences were found in the subsurface waters of

171 the northern stations within the Arabian Sea Oxygen Minimum Zone (Stations TARA_036 to

172 TARA_039), under the influence of a previous major bloom event, where high particle

173 concentrations and strong anaerobic microbial respiration have been reported ${ }^{26}$. Cluster 2

174 sequences were also found in lower abundance in the shallow anoxic zone of the Pacific

175 North Equatorial Counter Current (Stations TARA_137 and TARA_138, see methods). 


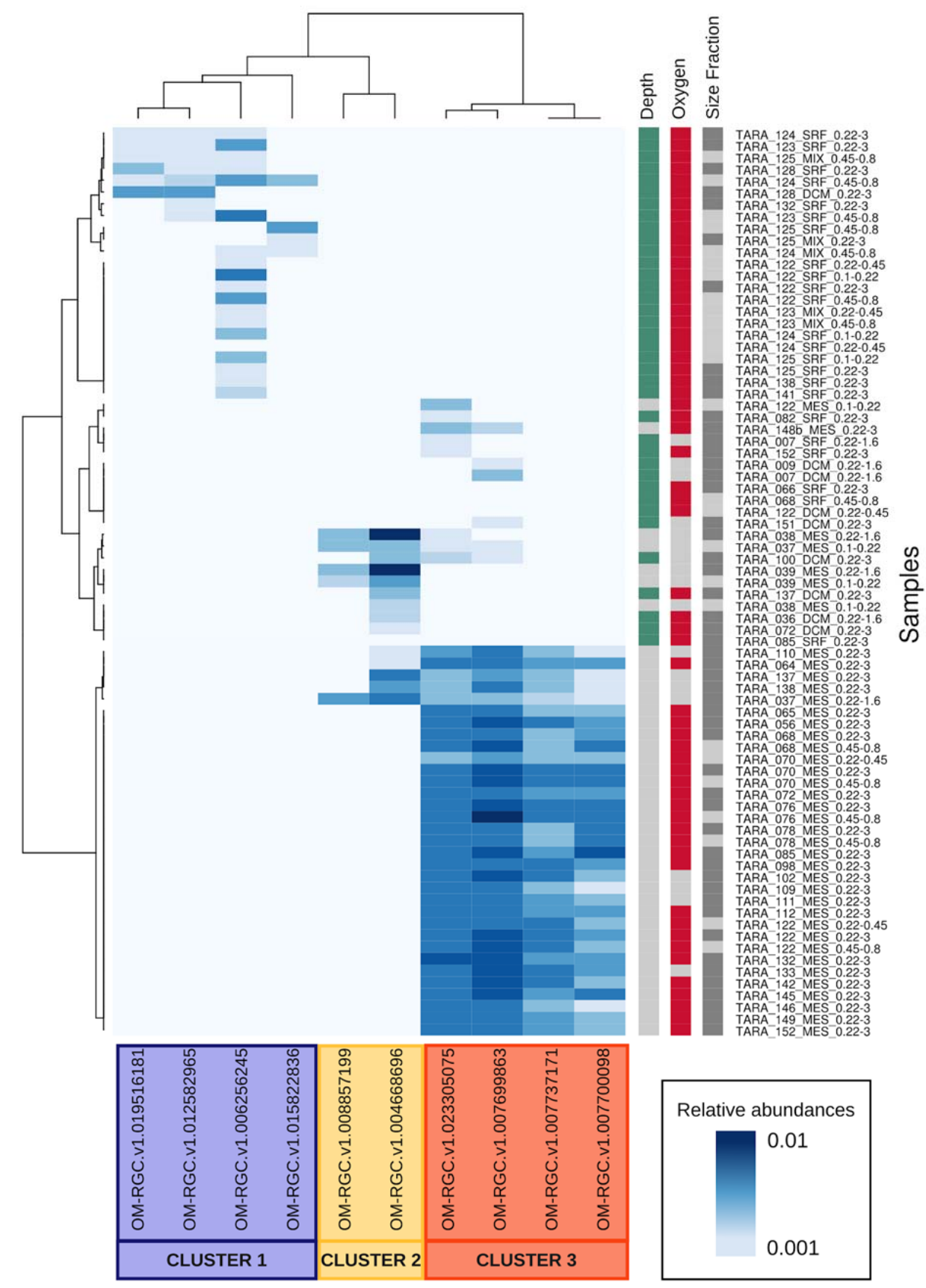

Figure 3 | Distribution of HgcA in Tara Oceans samples

178 HgcA relative abundance (from 0.01 to 0.1 ) is indicated by the white-blue gradient. The hierarchical

179 clustering highlighted three gene clusters with high abundances in specific samples with marked

180 environmental features, as suggested by colored squares. Surface samples were collected in the

181 upper layer (<120 m-depth, in green) while subsurface were collected below $120 \mathrm{~m}$-depth (in grey).

182 Seawater was considered as oxic when $\mathrm{O}_{2}>10 \mu \mathrm{M}$ (in red) and suboxic when $\mathrm{O}_{2}<10 \mu \mathrm{M}$ (in grey). 
183 Larger size fraction samples are in dark grey $(0.22-3 \mu \mathrm{m})$ and smaller size fractions samples $(<0.8 \mu \mathrm{m})$

184 are in light grey.

185

186

187 The most abundant HgcA-like proteins were grouped in Cluster 3 and were exclusively

188 assigned to Nitrospina. These Nitrospina HgcA-like proteins were found in 47 samples,

189 widespread across all sampled ocean basins. They were almost exclusively found in

190 subsurface water (> $120 \mathrm{~m}$-depth) and were more frequent in the oxic waters (> $\left.10 \mu \mathrm{M}_{\mathrm{O} 2}\right)$.

191 Subsurface oxic waters accounted for $84 \%$ of total Nitrospina-HgcA abundance

192 (Supplementary Figure 2). Their highest relative abundance was found in the South Atlantic

193 and the South Pacific Oceans (Fig. 4, Supplementary Table 2). Nitrospina HgcA abundance

194 was positively correlated to nitrate concentration ( $R 0.54, P<0.005)$, which is consistent

195 with Nitrospina's role as nitrate producer through nitrite oxidation, and with the well-known

196 nitrate enrichment with depth in the ocean.

198 Nitrospina as the most predominant and widespread methylator in the open ocean

199 The predominant and widespread HgcA-like homologs were phylogenetically extremely

200 close to the Nitrospina-related ones (Supplementary Figure 1) previously identified by

201 metagenomic analysis as potential Hg-methylators within Antarctic sea ice and brine, and

202 further detected by PCR in seawater samples below the ice ${ }^{13}$. The four Nitrospina HgcA-like

203 sequences from our study were distinct from HgcA in confirmed $\mathrm{Hg}$-methylators, and also

204 from HgcAB fusion proteins reported in environmental metagenomes ${ }^{12}$ (Figure 2). The few

205 cultured strains harboring a fused hgcAB gene (Methanococcoides methylutens and

206 Pyrococcus furiosus) were unable to produce MeHg in experimental conditions ${ }^{12,27}$. Through

207 sequence alignment against Protein Data Bank templates, we confirmed that the four 
208 Nitrospina HgcA-like homologs showed high conservation of six residue positions involved in

209 cobalamin binding, which is mandatory for methyl group transfer to $\mathrm{Hg}^{13}$ (Supplementary

210 Fig. 1). Protein structure modelling suggests that some Nitrospina species may be capable of

211 Hg-methylation. The observed mutations (N71 and C74) do not suppress Hg methylation

212 capacity, according to mutagenesis experiments in the model methylator $D$. desulfuricans

213 ND132 ${ }^{15}$. The strictly conserved cysteine facilitates the transfer of methyl groups to

214 inorganic $\mathrm{Hg}^{28}$. The two current Nitrospina isolates ( $N$. gracilis and $N$. watsonii) have not

215 been experimentally tested to date for their Hg-methylation capacity. $\mathrm{N}$. gracilis genome

216 lacks the $h g c A$ gene. The complete genome of $N$. watsonii is not available. From the 12

217 Nitrospina genome assemblies available on NCBI at the time of writing, we found HgcA-like

218 proteins (harbouring the six mandatory residues for $\mathrm{Hg}$-methylation) in three strains only:

219 SCGC AAA288-L16 (single cell whole genome from 770 m-deep ALOHA station, North Pacific

220 Ocean), AB-629-B06 (single cell whole genome from dark ocean water column) and LS_NOB

221 (from a deep-sea sponge holobiont; Supplementary Fig. 1).

222

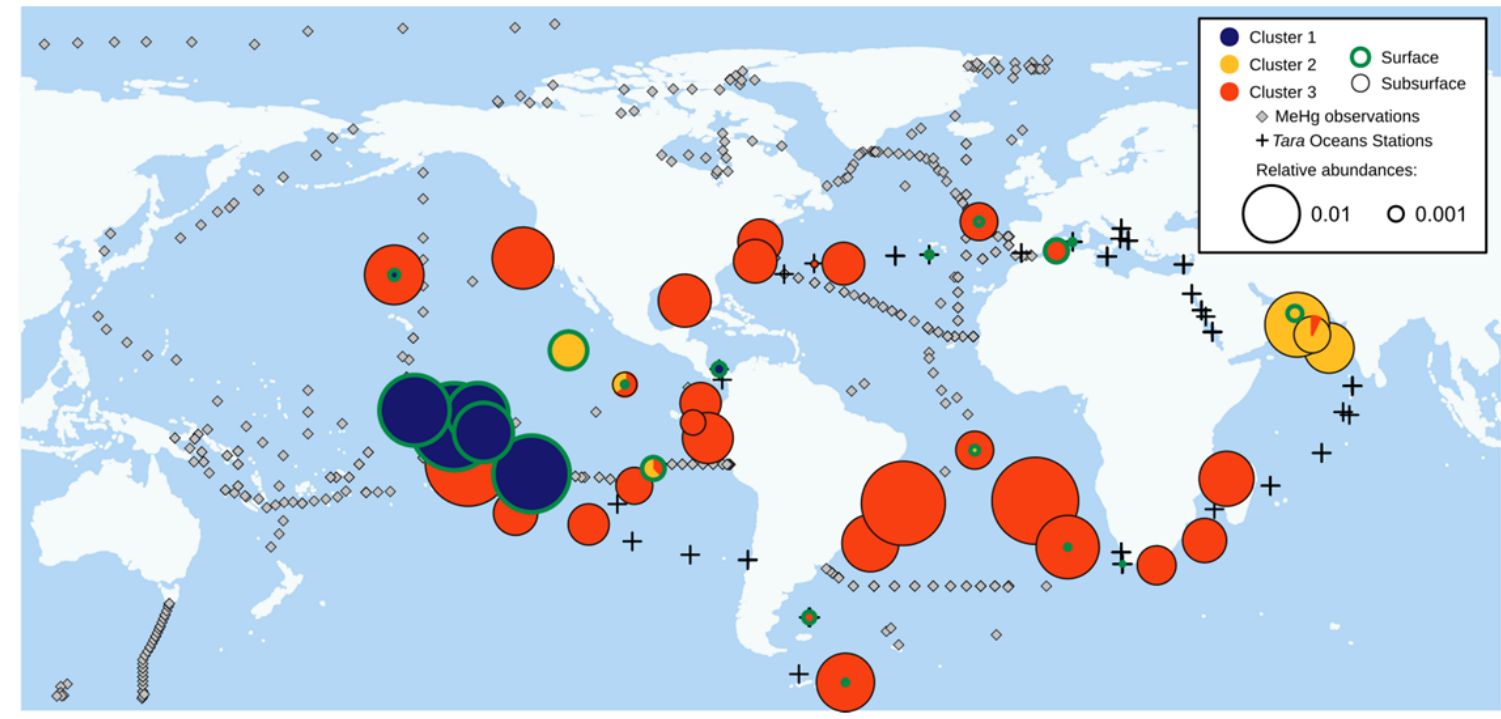

\section{Figure 4 | HgcA biogeography}

224 Circle sizes are proportional to the cumulated HgcA homolog genes abundances at each station. The 225 pie charts indicate the cluster attribution (legend in the chart), and their border color indicates the 
226 sampling depth: surface samples (<120 m-depth) in green and subsurface samples (>120 m-depth) in

227 grey. Tara Oceans stations without detected $\operatorname{HgcAB}$ genes are represented by black crosses and

228 seawater MeHg profiles from the literature (Supplementary Text 1) by black diamonds.

Mercury methylation has long been described for anaerobic environments ${ }^{12}$ and $h g c A$ genes

231 have been found exclusively in anaerobic Bacteria and Archea ${ }^{5}$. Yet, we find the most

232 abundant HgcA homologs are strongly dominant in oxic subsurface samples, where they

233 coincide with the subsurface MeHg concentration peaks ${ }^{6}$, and are carried by the nitrite

234 oxidizing bacteria Nitrospina, usually considered as aerobic.

235 Several clues may explain this apparent contradiction. First, it is increasingly recognized that

236 anaerobic processes can occur in anoxic niches such as organic matter aggregates in the

237 middle of oxic waters ${ }^{8}$. Nitrospina sequences were predominantly present in the larger size

238 fractions (accounting for $78 \%$ of total $\mathrm{HgcA}$ abundances), suggesting that $\mathrm{Hg}$-methylation -as

239 other anaerobic processes- might be associated with particles, where anoxic niches are likely

240 to be favourable to Nitrospina methylating activity. Several features suggest the adaptation

241 of Nitrospina to low-oxygen environments. Nitrospina have been detected as particularly

242 abundant (up to $10 \%$ of the bacterial community) in several upwelling and oxygen-deficient

243 zones $^{29}$.

244 Comparative genomics revealed a close evolutionary relationship between Nitrospina and

245 Anammox bacteria, including horizontal gene transfer events, suggesting the coexistence of

246 these organisms in hypoxic or anoxic environments ${ }^{16}$, as confirmed in incubation

247 experiments ${ }^{30,31}$. Genome analysis of several Nitrospina strains revealed unexpected

248 adaptation features to low-oxygen environments: no ROS defence mechanism, dependence

249 on highly oxygen-sensitive enzymes for carbon fixation, and high $\mathrm{O}_{2}$-affinity cytochromes

$250 \quad 16,32$ 
251 Nitrospina can play diverse ecological roles beyond the nitrogen cycle ${ }^{33}$. Nitrospina can use

252 alternative anaerobic pathways to gain energy, using other terminal electron acceptors than

$253 \mathrm{O}_{2}$ during fermentation under hypoxic or anaerobic conditions, such as sulfur compounds or

254 metal oxides. Their capacity to cope with environmental Hg through methylation is worth

255 considering, since their genome is well equipped against other toxic compounds (arsenate-

256 and mercuric-reductase, metallic cation transporters, multidrug export system) ${ }^{16,32}$.

257 Mercury methylation potential might have been acquired by horizontal gene transfer.

258 Within the four Nitrospina scaftigs harbouring $h g c A$, other neighbour genes related to

259 methyl group transfer and $\mathrm{Hg}$ metabolism are found, such as the merR1 regulator of the mer

260 operon involved in $\mathrm{Hg}$ resistance, the ubiE methyltransferase and the putative metal-binding

261 YHS domain (Fig. 1). This genomic context can lead to hypothesize that the expression of

262 these genes, including $h g c A$, is under the same $\mathrm{Hg}$-induced regulation as the mer operon,

263 triggered by merR.

264 The choice of $h g c A B$ as an indicator of Hg-methylation has to be discussed. First, the

265 presence of $h g c A B$ appears necessary but not sufficient for $\mathrm{Hg}$ methylation. Indeed,

266 unsuccessful attempts to transfer $\mathrm{Hg}$-methylation capacity to a non-Hg-methylating strain

267 suggest that unidentified additional genes might be needed for effective MeHg production

$268{ }^{15}$. Several critical steps are involved in the $\mathrm{Hg}$-methylation process, such as $\mathrm{Hg}(\mathrm{II})$ sensing,

269 cellular uptake of $\mathrm{Hg}(\mathrm{II})$ by active transport, methyl-group providing and transfer, and $\mathrm{MeHg}$

270 export from the cell. All these steps could be targeted as functional markers of $\mathrm{Hg}$ -

271 methylation in the environment in order to provide a more complete picture of the process.

272 Second, the exact contribution of $\mathrm{HgcAB}$ to $\mathrm{Hg}$-methylation is not well understood. Since $\mathrm{Hg}$

273 methylation does not confer $\mathrm{Hg}$ resistance, it cannot be considered as a protection

274 mechanism against $\mathrm{Hg}$ toxicity ${ }^{19}$. In model strains $D$. dechloroacetivorans, net $\mathrm{Hg}$ 
275 methylation was not clearly induced by inorganic $\mathrm{Hg}$ and not significantly correlated to

$276 h g c A B$ gene expression levels, but rather influenced by environmental factors, growth

277 conditions and energetic metabolism ${ }^{19,34}$. The variability of the methylation potential has

278 been evidenced in different strains, and the implication of hgcAB might also vary between

279 strains. Such functional gene approaches are powerful to track biogeochemical potentials in

280 extended environments but remain limited to well described metabolic pathways, ignoring

281 genes with unknown function ${ }^{35}$.

282 Here, we bring metagenomic evidence for widespread presence of microbial Hg-methylators

283 in the global ocean, thus reconciling with previous geochemical hints pointing to in situ

284 MeHg production in the water column. The key Hg-methylating genes found across all

285 oceans corresponded to taxonomic relatives of known Hg-methylators from

286 Deltaproteobacteria, Firmicutes and Chloroflexi phyla. We further identified the

287 microaerophilic NOB Nitrospina as the potential dominant $\mathrm{Hg}$-methylator in the global

288 ocean, ubiquitous at the DNA-level, and favoured by oxic subsurface waters (Supplementary

289 Figure 2). A critical next step would be to examine their hgcA expression levels and to

290 evaluate Hg-methylation capacity in Nitrospina cultures. Further studies should also

291 determine the physicochemical parameters controlling Nitropina Hg-methylation activity

292 level, in order to better understand how they will respond to expected global changes. Our

293 results open new avenues for disentangling the functional role of microorganisms in marine

$294 \mathrm{Hg}$ cycling. Our analysis of the Tara Oceans metagenomes reveals global distribution of the

295 key Hg methylating genes ( $h g c A$ and $h g c B$ ) and pinpoints Nitrospina as responsible for

296 widespread open ocean MeHg production in subsurface oxic seawater. Our study implicates

297 the subsurface oxic waters of all oceans as potential source of MeHg that should be

298 considered in the global Hg-cycle budgets, and identifies microbial target for further 
299 research on marine $\mathrm{MeHg}$ production. We hypothesize that besides anthropogenic $\mathrm{Hg}$

300 emissions, ongoing global climate change might have a previously underestimated effect on

301 in situ marine MeHg production by water-column microorganisms, by disturbing microbial

302 assemblages, activity, and environmental drivers governing Hg-methylation.

303 Methods

304 Identification of HgcAB environmental sequences in oceanic metagenomes.

$305 h g c A$ and $h g c B$ genes encode for a putative corrinoid protein, HgcA, and a 2[4Fe-4S]

306 ferredoxin, $\mathrm{HgcB}$, serving respectively as methyl group carrier and electron donor for

307 corrinoid cofactor reduction. HgcA and $\mathrm{HgcB}$ homologs were retrieved by searching Hidden

308 Markov Model profiles (HMM) ${ }^{36}$ provided by Podar et al. ${ }^{12}$ in the Ocean Microbial

309 Reference Gene Catalog ${ }^{14}$ (OM-RGC) using the Ocean Gene Atlas ${ }^{37}$ (http://tara-

310 oceans.mio.osupytheas.fr/ocean-gene-atlas/). The OM-RGC is the most exhaustive catalogue

311 of marine genes to date including datasets from Tara Oceans metagenomic assemblies and

312 other publicly available marine genomic and metagenomic datasets. We applied an e-value

313 threshold of 1e-20. The corresponding scaftigs (i.e. the assembled sequences where the

314 homolog genes were predicted) were retrieved from the raw assemblies deposited at ENA

315 (Supp Data 1 \& 4). Eight scaftigs without Tara Oceans mapped reads were discarded. The

316 remaining scaftigs were annotated using Prokka with default parameters ${ }^{38}$. The resulting

317 translated sequences were aligned separately for $\mathrm{HgcA}$ and $\mathrm{HgcB}$ using Jalview 2.10 and

318 alignments were cleaned manually ${ }^{39}$. For further analysis, we kept HgcA sequences if they

319 possess the conserved motif $\mathrm{NVWCAA}^{5}$, or if the neighbouring $\mathrm{HgcB}$ sequence was present

320 on the scaftig.

321 HgcA phylogenetic analysis. 
322 A phylogenetic tree was built from the $10 \mathrm{HgcA}$ sequences kept, $55 \mathrm{HgcA}$ protein sequences

323

324 representative of known Hg-methylator clades belonging to Archaea, Firmicutes, Chloroflexi and Deltaproteobacteria, including 18 experimentally-confirmed $\mathrm{Hg}$-methylators (initially published by Parks et al. ${ }^{5}$, and updated at https://www.esd.ornl.gov/programs/rsfa/data.shtml), as well as 9 HgcAB fusion proteins ${ }^{13}$ and 3 HgcA-like proteins predicted from Nitrospina genome assemblies using Prokka ${ }^{38}$. The tree was rooted with 3 paralogs from confirmed non-Hg-methylating strains ${ }^{13}$. The closest sequences (i.e. best e-value match) of each environmental HgcA sequence were retrieved using BLASTp against non-redundant RefSeq protein database excluding sequences from uncultured organisms ${ }^{40}$, and included in the tree.

The 80 sequences were aligned using MAFFT ${ }^{41}$ and gap-containing sites were removed using the mode gappyout of TrimAl ${ }^{42}$. Maximum likelihood phylogenies were inferred using PhyML Best AIC Tree (version 1.02b) implemented in Phylemon ${ }^{43}$ (version 2.0) with the best model of sequence evolution Blosum62+1+G+F. Branch support was calculated using the non-parametric Shimodaira-Hasegawa-like approximate likelihood ratio test. The final tree was edited using Evolview ${ }^{44}$, especially by annotating the isolation sites retrieved from Genomes OnLine Database ${ }^{45}$.

\section{Conserved sites in HgcA.}

Four sequences from OM-RGC related to Nitrospina were aligned with the same Protein

Data Bank (PDB) templates as Gionfriddo et al. ${ }^{13}$, as well as the 3 HgcA-like proteins from Nitrospina genome assemblies, and conserved residues were checked. The chosen PDB structural templates (4djd_C, 2h9a_A, 4C1n_C, 2ycl_A) were the gamma subunit of the corrinoid S-Fe acetyl-CoA decarbonylase/synthase complex, identified by Gionfriddo et al. ${ }^{13}$ as the closest and most complete relative to currently unresolved HgcA structure. 


\section{Biogeography of HgcA.}

347 Relative HgcA abundances in Tara Oceans samples were obtained from the Ocean Gene

348 Atlas $^{37}$. We screened 243 metagenomes from 68 sites covering the World Ocean except

349 Arctic, sampled at different depths from surface to 500 m-depth, covering six different size

350 fractions ranging from 0 to $3 \mu \mathrm{m}$. Environmental data were obtained from Pesant et al. ${ }^{46}$

351 (Supplementary Table 2). For the following analysis, we considered two depths classes

352 (surface samples $<120$ m-depth, subsurface samples $>120$ m-depth), two particle size

353 fractions $(<5 \mu \mathrm{m},<0.8 \mu \mathrm{m})$, two oxic states (oxic: $\mathrm{O}_{2}>10 \mu \mathrm{M}$, suboxic: $\mathrm{O}_{2}<10 \mu \mathrm{M}$ ).

354 HgcA relative abundance was calculated as follows: the length-normalized count of genes

355 read was divided by the median of the length-normalized counts of a set of ten universal

356 single copy marker genes ${ }^{47,48}$. Thus, relative abundance represents the fraction of bacteria

357 harbouring the $h g c A$ gene within the assembled genomes. A heatmap of the relative gene

358 abundances in Tara Oceans samples was generated in $\mathrm{R}^{49}$ using the heatmap. 2 function in

359 the ggplot CRAN library. Dendrograms were computed using hclust default parameters from

360 Ward distance index based on presence/absence of the genes ('binary' option). Genes were

361 clustered into three groups (Cluster 1, Cluster 2 and Cluster 3) according to their abundance

362 pattern on the heatmap. The geographic origin of the hgcA genes retrieved from the Tara

363 Oceans samples was plotted on a global map using the "mapplots" R package. At each

364 station, the cumulated abundance and phylogenetic affiliation of the retrieved $h g c A$ genes

365 were represented on the map by the size and colour of the points. Cluster distribution was

366 also plotted against depth and oxygen concentration at each station to depict the

367 environmental conditions where each Cluster flourishes (Supplementary Figure 2). Tracks of

368 MeHg records from previous campaigns were searched in the literature (Supplementary Text

369 1) and georeferenced on the map. 
371 References

372 1. Outridge, P. M., Mason, R. P., Wang, F., Guerrero, S. \& Heimbürger-Boavida, L. E.

373 Updated Global and Oceanic Mercury Budgets for the United Nations Global Mercury

374 Assessment 2018. Environ. Sci. Technol. 52, 11466-11477 (2018).

375 2. Gilmour, C. C. et al. Mercury methylation by novel microorganisms from new

376 environments. Environ. Sci. Technol. 47, 11810-11820 (2013).

377 3. Gilmour, C. C. et al. Sulfate-Reducing Bacterium Desulfovibrio desulfuricans ND132 as 378 a Model for Understanding Bacterial Mercury Methylation. Appl. Environ. Microbiol.

$379 \quad 77,3938-3951(2011)$.

380

4. Mason, R. P. et al. Mercury biogeochemical cycling in the ocean and policy

381 implications. Environ. Res. 119, 101-117 (2012).

382

5. Parks, J. M. et al. The genetic basis for bacterial mercury methylation. Science (80-. ). 339, 1332-1335 (2013).

6. Schlitzer, R. et al. The GEOTRACES Intermediate Data Product 2017. Chem. Geol. 493, 210-223 (2018). mercury and methylmercury particulate distributions, methylation and demethylation rates in laboratory-generated marine snow. Mar. Chem. 177, 753-762 (2015). 
389 8. Bianchi, D., Weber, T. S., Kiko, R. \& Deutsch, C. Global niche of marine anaerobic 390 metabolisms expanded by particle microenvironments. Nat. Geosci. 1-6 (2018). doi:10.1038/s41561-018-0081-0

392

9. Lehnherr, I., St. Louis, V. L., Hintelmann, H. \& Kirk, J. L. Methylation of inorganic mercury in polar marine waters. Nat. Geosci. 4, 298-302 (2011).

10. Blum, J. D. Marine mercury breakdown. Nat. Geosci. 4, 139-140 (2011).

395

11. Masbou, J. et al. Carbon Stable Isotope Analysis of Methylmercury Toxin in Biological Materials by Gas Chromatography Isotope Ratio Mass Spectrometry. Anal. Chem. 87, $11732-11738$ (2015).

12. Podar, M. et al. Global prevalence and distribution of genes and microorganisms involved in mercury methylation. Sci. Adv. 1, (2015).

13. Gionfriddo, C. M. et al. Microbial mercury methylation in Antarctic sea ice. Nat. Microbiol. 1, 16127 (2016).

14. Sunagawa, S. et al. Ocean plankton. Structure and function of the global ocean microbiome. Science 348, 1261359 (2015).

15. Smith, S. D. et al. Site-directed mutagenesis of $\mathrm{HgcA}$ and $\mathrm{HgcB}$ reveals amino acid residues important for mercury methylation. Appl. Environ. Microbiol. 81, 3205-17 (2015).

16. Lücker, S., Nowka, B., Rattei, T., Spieck, E. \& Daims, H. The Genome of Nitrospina gracilis Illuminates the Metabolism and Evolution of the Major Marine Nitrite 
Oxidizer. Front. Microbiol. 4, 27 (2013).

410 17. Spieck, E., Keuter, S., Wenzel, T., Bock, E. \& Ludwig, W. Characterization of a new marine nitrite oxidizing bacterium, Nitrospina watsonii sp. nov., a member of the newly proposed phylum “Nitrospinae”. Syst. Appl. Microbiol. 37, 170-176 (2014).

18. Cao, J. et al. Pseudodesulfovibrio indicus gen. nov., sp. nov., a piezophilic sulfatereducing bacterium from the Indian Ocean and reclassification of four species of the genus Desulfovibrio. Int. J. Syst. Evol. Microbiol. 66, 3904-3911 (2016). a model for understanding bacterial mercury methylation. Appl. Environ. Microbiol. 77, 3938-51 (2011).

20. Bae, H.-S., Dierberg, F. E. \& Ogram, A. Syntrophs dominate sequences associated with the mercury methylation-related gene hgcA in the water conservation areas of the Florida Everglades. Appl. Environ. Microbiol. 80, 6517-26 (2014).

21. Sorokin, D. Y., Tourova, T. P., Mußmann, M. \& Muyzer, G. Dethiobacter alkaliphilus gen. nov. sp. nov., and Desulfurivibrio alkaliphilus gen. nov. sp. nov.: two novel representatives of reductive sulfur cycle from soda lakes. Extremophiles 12, 431-439 (2008). 
Critical Review of Published Field and Laboratory Studies. Environ. Sci. Technol. 53, 419 (2019).

431

432

433

434

435

436

437

438

439

440

441

442

443

444

445

446

447

24. Sela-Adler, M. et al. Co-existence of Methanogenesis and Sulfate Reduction with Common Substrates in Sulfate-Rich Estuarine Sediments. Front. Microbiol. 8, 766 (2017).

25. Pak, K. R. \& Bartha, R. Mercury methylation by interspecies hydrogen and acetate transfer between sulfidogens and methanogens. Appl. Environ. Microbiol. 64, 19871990 (1998).

26. Roullier, F. et al. Particle size distribution and estimated carbon flux across the Arabian Sea oxygen minimum zone. Biogeosciences 11, 4541-4557 (2014).

27. Gilmour, C. C., Bullock, A. L., McBurney, A., Podar, M. \& Elias, D. A. Robust mercury methylation across diverse methanogenic Archaea. MBio 9, (2018).

28. Zhou, J., Riccardi, D., Beste, A., Smith, J. C. \& Parks, J. M. Mercury Methylation by HgcA: Theory Supports Carbanion Transfer to Hg(II). Inorg. Chem. 53, 772-777 (2014).

29. Levipan, H. A., Molina, V. \& Fernandez, C. Nitrospina-like bacteria are the main drivers of nitrite oxidation in the seasonal upwelling area of the Eastern South Pacific (Central Chile $\sim 36^{\circ}$ S). Environ. Microbiol. Rep. 6, 565-573 (2014).

30. Füssel, J. et al. Nitrite oxidation in the Namibian oxygen minimum zone. ISME J. 6, 1200-1209 (2012). 
449

450

451

452

453

454

455

456

457

458

459

460

461

462

463

464

465

466

and oxygen minimum zone of the eastern tropical North Pacific Ocean. ISME J. 7, 2192-2205 (2013).

32. Ngugi, D. K., Blom, J., Stepanauskas, R. \& Stingl, U. Diversification and niche adaptations of Nitrospina-like bacteria in the polyextreme interfaces of Red Sea brines. ISME J. 10, 1383-1399 (2016).

33. Daims, H., Lücker, S. \& Wagner, M. A New Perspective on Microbes Formerly Known as Nitrite-Oxidizing Bacteria. Trends Microbiol. 24, 699-712 (2016).

34. Goñi-Urriza, M. et al. Relationships between bacterial energetic metabolism, mercury methylation potential, and hgcA/hgcB gene expression in Desulfovibrio dechloroacetivorans BerOc1. Environ. Sci. Pollut. Res. 22, 13764-13771 (2015).

35. Reed, D. C., Algar, C. K., Huber, J. A. \& Dick, G. J. Gene-centric approach to integrating environmental genomics and biogeochemical models. Proc. Natl. Acad. Sci. U. S. A. 111, 1879-84 (2014).

36. Eddy, S. R. Accelerated profile HMM searches. PLoS Comput. Biol. 7, e1002195 (2011).

37. Villar, E. et al. The Ocean Gene Atlas: exploring the biogeography of plankton genes online. Nucleic Acids Res. 46, W289-W295 (2018).

38. Seemann, T. Prokka: rapid prokaryotic genome annotation. Bioinformatics 30, 20682069 (2014).

39. Waterhouse, A. M., Procter, J. B., Martin, D. M. A., Clamp, M. \& Barton, G. J. Jalview Version 2-a multiple sequence alignment editor and analysis workbench. 
Bioinformatics 25, 1189-1191 (2009).

470

471

472

473

474

475

476

477

478

479

480

481

482

483

484

485

486

487

488

40. Altschul, S. F., Gish, W., Miller, W., Myers, E. W. \& Lipman, D. J. Basic local alignment search tool. J. Mol. Biol. 215, 403-410 (1990).

41. Katoh, K. \& Standley, D. M. MAFFT multiple sequence alignment software version 7: improvements in performance and usability. Mol. Biol. Evol. 30, 772-780 (2013).

42. Capella-Gutierrez, S., Silla-Martinez, J. M. \& Gabaldon, T. trimAl: a tool for automated alignment trimming in large-scale phylogenetic analyses. Bioinformatics 25, 19721973 (2009).

43. Sanchez, R. et al. Phylemon 2.0: a suite of web-tools for molecular evolution, phylogenetics, phylogenomics and hypotheses testing. Nucleic Acids Res. 39, W470W474 (2011).

44. Gao, S. et al. Evolview v2: an online visualization and management tool for customized and annotated phylogenetic trees. Nucleic Acids Res. 44, W236-W241 (2016).

45. Mukherjee, S. et al. Genomes OnLine Database (GOLD) v.6: data updates and feature enhancements. Nucleic Acids Res. 45, D446-D456 (2017).

46. Pesant, S. et al. Open science resources for the discovery and analysis of Tara Oceans data. Sci. Data 2, 150023 (2015).

47. Mende, D. R., Sunagawa, S., Zeller, G. \& Bork, P. Accurate and universal delineation of prokaryotic species. Nat. Methods 10, 881-884 (2013). 
489 48. Sunagawa, S. et al. Metagenomic species profiling using universal phylogenetic

490 marker genes. Nat. Methods 10, 1196-1199 (2013).

491

49. R Development Core Team. R: A Language and Environment for Statistical Computing.

492 (2008).

493

494

495 Acknowledgements

496 The authors thank Patricia Bonin, Joana R.H. Boavida, Pascal Hingamp, Eric Pelletier, Daniel

497 Cossa, Jeroen E. Sonke for constructive comments that helped to improve this manuscript.

$498 \quad$ Funding

499 E.V. received funding from the project IMPEKAB ANR-15-CE02-0011

500 Author contributions

501 E.V., L.C. and L.E.H.B. wrote the manuscript. E.V. performed the bioinformatic analyses with

502 the scientific support of L.C.

503

504 